\title{
Morbidity, mortality, and obesity paradox
}

\author{
Michael Spartalis ${ }^{1}$, Eleni Tzatzaki ${ }^{1}$, Demetrios Moris ${ }^{2}$, Antonios Athanasiou ${ }^{3}$, Eleftherios Spartalis ${ }^{4}$ \\ ${ }^{1}$ Division of Cardiology, Onassis Cardiac Surgery Center, Athens, Greece; ${ }^{2}$ Department of Surgery, The Ohio State University Comprehensive \\ Cancer Center, The Ohio State University, Columbus, OH, USA; ${ }^{3}$ Department of Surgery, Mercy University Hospital, Cork, Ireland; ${ }^{4}$ Laboratory \\ of Experimental Surgery and Surgical Research, University of Athens Medical School, Athens, Greece \\ Correspondence to: Demetrios Moris, MD, PhD. Department of Surgery, The Ohio State University Comprehensive Cancer Center, The Ohio State \\ University, Columbus, OH, USA. Email: dimmoris@yahoo.com.
}

Submitted Aug 04, 2017. Accepted for publication Aug 15, 2017.

doi: $10.21037 / \mathrm{atm} .2017 .08 .31$

View this article at: http://dx.doi.org/10.21037/atm.2017.08.31

We read with great interest the article by Abdelaal et al. (1) titled "Morbidity and mortality associated with obesity". The authors state that the prevalence and disease burden of high body mass index (BMI) are increasing. The escalating prevalence of obesity and its link to substantial increase in metabolic and cardiovascular disease is a known fact (1). Current studies and meta-analyses, though, demonstrate with consistent effects, the presence of an "obesity paradox" among patients with several diseases such as coronary artery disease, chronic kidney disease, heart failure, atrial fibrillation, diabetes and patients undergoing cardiac surgery (2-10). This obesity paradox shows that lower (rather than higher) BMI is a risk factor for increased mortality (2-10).

A recent study of 345,192 patients from the British Cardiovascular Intervention Society registry examined the relationship between BMI and clinical outcomes following percutaneous coronary intervention (PCI) (2). The study concluded that there was an independent association of elevated BMI with reduced mortality after PCI, both in stable and acute clinical settings (2).

The ARIC study investigated 1,279 individuals and examined the association of pre-heart failure BMI with mortality after incident heart failure hospitalization (3). The study resulted that, in comparison to normal weight subjects the mortality was lower in overweight (3). Obesity increases the risk for development of heart failure, but it appears to exert a protective effect in patients in whom heart failure has already been diagnosed (4).

A recent meta-analysis of 401,227 adult patients undergoing cardiac surgery reported that obesity is associated with lower mortality risk after cardiac surgery, with consistent effects noted in multiple analyses attempting to address residual confounding and reverse causation (5). Hartrumpf et al. analyzed data from 15,314 consecutive patients receiving major cardiac surgery and also confirmed the obesity paradox showing the least mortality in the overweight group (6).

Proietti et al. performed a systematic review on the relationship between BMI and major adverse outcomes in atrial fibrillation patients (7). Obesity paradox was evident in atrial fibrillation patients, particularly for all-cause and cardiovascular death outcomes. A significant obesity paradox was also found with both overweight and obese patients reporting a lower risk for stroke/systemic embolic event (7).

Kwon et al. conducted a systematic review and meta-analysis of sixteen studies, in order to quantify the association between BMI and the risk of all-cause and cardiovascular mortality in patients with type 2 diabetes (8). The authors resulted that an obesity paradox also exists for patients with type 2 diabetes concerning allcause and cardiovascular mortality (8).

Furthermore, obesity confers survival advantages in advanced chronic kidney disease (9). A retrospective study by Tsang et al. investigated the potential association between BMI and overall survival in patients with distant metastases (10). Compared with normal-weight metastatic patients, both obese and overweight individuals had a reduced risk of all-cause mortality (10).

Different mechanisms have been reported that may be responsible for this paradox. These mechanisms are hemodynamic stability of obesity, lipoprotein defense against circulating endotoxins, protective cytokine profiles, 
toxin sequestration of fat mass, and antioxidation of muscle may (9).

The phenomenon of obesity paradox is in sharp contradistinction to the known epidemiology of cardiovascular risks. The direct effects of obesity on cardiovascular function and health require further exploration, and the investigation of the causes and consequences of the obesity paradox can identify the pathophysiologic mechanisms leading to improved outcomes.

\section{Acknowledgements}

None.

\section{Footnote}

Conflicts of Interest: The authors have no conflicts of interest to declare.

\section{References}

1. Abdelaal M, le Roux CW, Docherty NG. Morbidity and mortality associated with obesity. Ann Transl Med 2017;5:161.

2. Holroyd EW, Sirker A, Kwok CS, et al. The Relationship of Body Mass Index to Percutaneous Coronary Intervention Outcomes: Does the Obesity Paradox Exist in Contemporary Percutaneous Coronary Intervention Cohorts? Insights From the British Cardiovascular Intervention Society Registry. JACC Cardiovasc Interv

Cite this article as: Spartalis $M$, Tzatzaki E, Moris D, Athanasiou A, Spartalis E. Morbidity, mortality, and obesity paradox. Ann Transl Med 2017;5(21):440. doi: 10.21037/ atm.2017.08.31
2017;10:1283-92.

3. Pokharel Y, Sun W, Virani SS, et al. Myocardial Injury, Obesity, and the Obesity Paradox: The ARIC Study. JACC Heart Fail 2017;5:56-63.

4. Carbone S, Lavie CJ, Arena R. Obesity and Heart Failure: Focus on the Obesity Paradox. Mayo Clin Proc 2017;92:266-79.

5. Mariscalco G, Wozniak MJ, Dawson AG, et al. Body Mass Index and Mortality Among Adults Undergoing Cardiac Surgery: A Nationwide Study With a Systematic Review and Meta-Analysis. Circulation 2017;135:850-63.

6. Hartrumpf M, Kuehnel RU, Albes JM. The obesity paradox is still there: a risk analysis of over 15000 cardiosurgical patients based on body mass index. Interact Cardiovasc Thorac Surg 2017;25:18-24.

7. Proietti M, Guiducci E, Cheli P, et al. Is There an Obesity Paradox for Outcomes in Atrial Fibrillation? A Systematic Review and Meta-Analysis of Non-Vitamin K Antagonist Oral Anticoagulant Trials. Stroke 2017;48:857-66.

8. Kwon Y, Kim HJ, Park S, et al. Body Mass IndexRelated Mortality in Patients with Type 2 Diabetes and Heterogeneity in Obesity Paradox Studies: A DoseResponse Meta-Analysis. PLoS One 2017;12:e168247.

9. Kalantar-Zadeh K, Rhee CM, Chou J, et al. The Obesity Paradox in Kidney Disease: How to Reconcile it with Obesity Management. Kidney Int Rep 2017;2:271-81.

10. Tsang NM, Pai PC, Chuang CC, et al. Overweight and obesity predict better overall survival rates in cancer patients with distant metastases. Cancer Med 2016;5:665-75. 